\title{
Can sequence learning be implicit? New evidence with the process dissociation procedure
}

\author{
ARNAUD DESTREBECQZ and AXEL CLEEREMANS \\ Université Libre de Bruxelles, Brussels, Belgium
}

\begin{abstract}
Can we learn without awareness? Although this issue has been extensively explored through studies of implicit learning, there is currently no agreement about the extent to which knowledge can be acquired and projected onto performance in an unconscious way. The controversy, like that surrounding implicit memory, seems to be at least in part attributable to unquestioned acceptance of the unrealistic assumption that tasks are process-pure - that is, that a given task exclusively involves either implicit or explicit knowledge. Methods such as the process dissociation procedure (PDP, Jacoby, 1991) have been developed to overcome the conceptual limitations of the process purity assumption but have seldom been used in the context of implicit learning research. In this paper, we show how the PDP can be applied to a free generation task so as to disentangle explicit and implicit sequence learning. Our results indicate that subjects who are denied preparation to the next stimulus nevertheless exhibit knowledge of the sequence through their reaction time performance despite remaining unable (1) to project this knowledge in a recognition task and (2) to refrain from expressing their knowledge when specifically instructed to do so. These findings provide strong evidence that sequence learning can be unconscious.
\end{abstract}

The role that consciousness plays in cognition is one of the most central and long-standing issues in experimental psychology. Differences between conscious and unconscious processing have indeed been explored in many different fields, such as memory (e.g., Jacoby, 1991; see also Kinoshita, 2001), perception (Reingold \& Merikle, 1988), conditioning (Clark \& Squire, 1998; Lieberman, Sunnucks, \& Kirk, 1998), and learning (e.g., Cleeremans, Destrebecqz, \& Boyer, 1998; Shanks \& St. John, 1994). Today, these issues benefit from renewed and widespread interest in the study of consciousnessperhaps best exemplified by the "search for the neural correlates of consciousness" now made possible by the increased availability of brain imaging techniques (see Frith, Perry, \& Lumer, 1999, for a review).

Despite this wealth of research, the role of consciousness in learning remains intensely controversial (Stadler \& Roediger, 1998), with some authors concluding that "human learning is systematically accompanied by awareness" (Shanks \& St. John, 1994, p. 394), and others ar-

A.D. is a Scientific Research Worker of the National Fund for Scientific Research (Belgium). A.C. is a Research Associate of the National Fund for Scientific Research (Belgium). This research was also supported by a grant from the Université Libre de Bruxelles to A.C. in support of IUAP Program P/4-19. We thank Robert M. French, Larry Jacoby, Pierre Perruchet, David Shanks, Tim Curran, Arthur S. Reber, John T. Wixted, and an anonymous reviewer for crucial suggestions and many useful comments on a previous version of this paper. Correspondence should be addressed to A. Destrebecqz, Cognitive Science Research Unit, Université Libre de Bruxelles, Ave. F. D. Roosevelt, 50-CP 122, 1050 Brussels, Belgium (e-mail: adestre@ulb.ac.be). guing that unconscious learning is a fundamental process in human cognition (Reber, 1993). In this paper, we demonstrate that learning can be unconscious to the extent that the relevant knowledge can influence behavior yet remain unavailable to conscious control. To do so, we apply one of the better methods of assessing awarenessthe process dissociation procedure (PDP) introduced by Jacoby (1991) - to the best paradigm through which to study implicit learning - that is, sequence learning.

In a typical sequence learning situation (see Clegg, DiGirolamo, \& Keele, 1998), subjects are asked to react to each element of a sequentially structured and typically visual sequence of events in the context of a serial reaction time (SRT) task. On each trial, subjects see a stimulus appear at one of several locations on a computer screen and are asked to press as fast and as accurately as possible on the corresponding key. Unbeknownst to them, the sequence of successive stimuli follows a repeating pattern (Nissen \& Bullemer, 1987). Reaction times (RTs) tend to decrease progressively during practice but to increase dramatically when the repeating pattern is modified in any of several ways (Cohen, Ivry, \& Keele, 1990; Curran \& Keele, 1993; Reed \& Johnson, 1994). This finding suggests that subjects have learned the pattern and are able to prepare their responses on the basis of their knowledge of the sequence. Nevertheless, subjects often fail to exhibit verbalizable knowledge of the pattern (Curran \& Keele, 1993; Willingham, Nissen, \& Bullemer, 1989)—a dissociation that has led many authors to consider learning in this situation to be implicit.

Implicit learning has received many different operational definitions, but the most neutral and commonly ac- 
cepted one simply states that learning is implicit when we acquire new information in such a way that the resulting knowledge is difficult to express (Berry \& Dienes, 1993). Accordingly, most empirical studies of implicit learning have taken the form of dissociation paradigms on the basis of the rationale that in order to demonstrate implicit learning, it is sufficient to show that learning is not accompanied by awareness, as assessed by a subsequent test of explicit knowledge.

Such dissociations have often been obtained between performance in the SRT task and sequence knowledge as expressed in verbal reports. However, Shanks and St. John (1994) have convincingly argued that verbal reports do not constitute sufficiently sensitive tests of explicit knowledge, and that the corresponding dissociation findings should therefore be rejected as valid demonstrations of implicit learning. Indeed, verbal reports could fail to detect knowledge held with low confidence or could probe subjects about knowledge that they do not even need in order to perform the task (e.g., knowledge of rules when knowledge of instances is sufficient). As a result, Shanks and St. John, as well as many other authors (e.g., Jiménez Méndez \& Cleeremans, 1996; Perruchet \& Amorim, 1992), have suggested that valid tests of explicit sequence knowledge should involve forced-choice tasks, such as generation or recognition. ${ }^{1}$

With a few exceptions that turned out not to be immune from methodological concerns (see Shanks \& Johnstone, 1998), all sequence learning studies using forced-choice tests have demonstrated strong associations between performance on such tests and learning as measured through the SRT task. These findings have prompted many authors to conclude that there is in fact no or very little evidence for implicit sequence learning (Shanks \& Johnstone, 1999). This conclusion seems to be at odds with studies showing relatively preserved learning capacities in memory-impaired subjects (see Curran, 1995, for a review). However, only a few such studies have been reported so far. Furthermore, existing results are partly contradictory and often remain open to different interpretations. For instance, according to Shanks and colleagues (Shanks \& Johnstone, 1999; Shanks \& St. John, 1994), amnesics' performance cannot be taken as a demonstration of implicit learning because, when compared with that of control subjects, their performance is generally not only impaired in forced-choice tasks (taken as an index of explicit knowledge), but also in the SRT task (in which performance is assumed to reflect implicit sequence learning).

Importantly, these debates all tend to be rooted in the questionable assumption that tasks are "process-pure." In this paper, we defend a different position, namely that successful performance on forced-choice tasks cannot be exclusively attributed to the influence of explicit knowledge. In a generation task, for instance, subjects could perform above chance when they believed they were guessing the location of the next stimulus (Shanks \& Johnstone, 1998). Likewise, in a recognition task, they could respond on the basis of a feeling of familiarity in the absence of explicit recollection of the sequence (see Reber, Allen, \& Reagan, 1985, and Cohen \& Curran, 1993, for further discussion). Implicit learning research is therefore cornered in a difficult methodological dilemma, because the most sensitive tests of explicit knowledge also turn out to be those most likely to be contaminated by implicit knowledge (Neal \& Hesketh, 1997).

Similar issues raised in the implicit memory and subliminal perception literatures have fostered the development of new methodologies that assume that tasks in general are not process-pure (Merikle \& Reingold, 1991; Reingold \& Merikle, 1988). For instance, Jacoby (1991) has proposed the PDP as a way to establish the existence of qualitative dissociations between explicit and implicit forms of memory. In stem-completion tasks, for instance (see Jacoby, Toth, \& Yonelinas, 1993), the number of word stems completed with previously studied words is compared in two conditions: the inclusion condition, in which subjects are asked to use either studied words to complete the stems or, failing recollection, the first word that comes to mind, and the exclusion condition, in which subjects are asked to exclude studied words when completing the stems. If studied completions are nevertheless produced in the exclusion condition, such responses can only be interpreted as reflecting the implicit influence of memorized items. ${ }^{2}$

Adaptations of the PDP to sequence learning (Buchner, Steffens, Erdfelder, \& Rothkegel, 1997; Buchner, Steffens, \& Rothkegel, 1998; see also Goschke, 1997, 1998) and artificial grammar learning (Dienes, Altmann, Kwan, \& Goode, 1995) have been previously reported. Buchner and colleagues used the procedure to differentiate between explicit recollection and perceptual fluency in a recognition task subsequent to an auditory version of the SRT task. However, as stated before by Shanks and Johnstone (1999), the perceptual-motor fluency effect cannot be considered as equivalent to implicit influence given that fluency is consciously experienced and that it may be associated with explicit sequence knowledge.

In this paper, we propose a novel adaptation of the PDP to sequence learning in order to disentangle implicit and explicit knowledge acquisition in the SRT task. To assess explicit knowledge after training on the SRT task, we used a so-called "free generation" task-previously shown to be a very sensitive test of sequence knowledge (Perruchet \& Amorim, 1992)—and asked subjects to perform this task under both inclusion and exclusion instructions (see below).

Next, to manipulate the extent to which sequence learning is explicit, we hypothesized that response preparation in the SRT task always involves both implicit and explicit components, and that the latter are specifically sensitive to the duration of the response-stimulus interval (RSI) - that is, the interval that separates subjects' responses and the onset of the next stimulus. In other words, our main hypothesis was that the development of explicit knowledge of the sequence depends on the rate of stimulus presentation in the SRT task. On the basis of 
this hypothesis, we thus compared subjects' performance in two conditions differing only in terms of RSI: In the no-RSI condition, this delay was eliminated in order to prevent explicit preparation to the onset of the next stimulus. In the RSI condition, the RSI was set to a standard $250 \mathrm{msec}$. Our hypothesis was that suppressing the RSI would exclusively impair explicit sequence learning (but see also Perruchet, Bigand, \& Benoît-Gonin, 1997, for a different position). If this were confirmed, we would expect to find that, in contrast to subjects trained in the RSI condition, subjects trained in the no-RSI condition acquire knowledge that, when assessed through our direct tests, would appear to be essentially implicit.

\section{METHOD}

\section{Subjects}

Twenty-four subjects aged 18-26 years, all undergraduate students at the Université Libre de Bruxelles, were randomly assigned to one of two experimental conditions and paid $\$ 10$.

\section{Materials}

The experiment was run on Macintosh computers. The display consisted of four dots arranged in a horizontal line on the computer's screen and separated by intervals of $3 \mathrm{~cm}$. Each screen position corresponded to a key on the computer's keyboard. The spatial configuration of the keys was fully compatible with the screen positions. The stimulus was a small black circle $0.35 \mathrm{~cm}$ in diameter that appeared on a white background, centered $1 \mathrm{~cm}$ above one of the four dots.

\section{Procedure}

The experiment consisted of 15 training blocks during which subjects were exposed to a serial four-choice RT task. Each block consisted of 96 trials for a total of 1,440 trials. On each trial, a stimulus appeared at one of the four possible screen locations. Subjects were instructed to respond as fast and as accurately as possible by pressing the corresponding key. The target was removed as soon as a key had been pressed, and the next stimulus appeared after either a 0 -msec (no-RSI condition) or a 250 -msec (RSI condition) interval depending on the condition. Erroneous responses were signaled to subjects by means of a tone. Short rest breaks occurred between any two experimental blocks. Subjects were presented with one of the following 12-element sequences: 342312143241 (SOC1) or 341243142132 (SOC2). Each experimental block consisted of eight repetitions of the sequence. These sequences consisted entirely of so-called "second order conditional" transitions (SOCs; Reed \& Johnson, 1994). With SOC sequences, two elements of temporal context are always necessary to predict the location of the next stimulus. Both sequences were balanced for stimulus locations and transition frequency but differed in terms of the subsequence of three elements that they contained. For instance, the transition 34 was followed by Location 2 in SOC1 and by Location 1 in SOC2. In each condition, half the subjects were trained on SOC1 during the first 12 blocks and during Blocks 14 and 15, and on SOC2 during Block 13. This design was reversed for the other half of the subjects. Increased RTs during Block 13 are thus expected only if subjects have acquired SOC knowledge during training over Blocks 1-12.

After the SRT task, subjects were informed that the dots had followed a repeating pattern. They were then presented with a single stimulus that appeared in a random location and asked to freely generate a series of 96 trials that "resembled the training sequence as much as possible." They were told to rely on their intuitions when feeling unable to recollect the location of the next stimulus. After this generation task-performed under inclusion instructionssubjects were asked to generate another sequence of 96 trials, this time under exclusion instructions. They were told they now had to try to avoid reproducing the sequential regularities of the training sequences. In both generation tasks, subjects were also told not to repeat responses. The stimulus moved whenever subjects had pressed one of the keys, and appeared at the corresponding location after a delay of either 0 or $250 \mathrm{msec}$, depending on the condition.

After completion of the two generation tasks, subjects were asked to perform a recognition task. Here, we used a procedure identical to that described in Shanks and Johnstone (1999). Subjects were presented with 24 fragments of three trials. Twelve were part of SOC1, and 12 were part of SOC2. Subjects were asked to respond to the stimuli as in the SRT task, and then to provide a rating of how confident they were that the fragment was part of the training sequence. Ratings involved a 6-point scale ( $1=I$ 'm certain that this fragment was part of the training sequence, $2=I$ ' $m$ fairly certain that this fragment was part of the training sequence, $3=$ I believe that this fragment was part of the training sequence, $4=$ I believe that this fragment was not part of the training sequence, $5=I$ ' $m$ fairly certain that this fragment was not part of the training sequence, and $6=I^{\prime} m$ certain that this fragment was not part of the training sequence. It was emphasized to subjects that they had to respond as fast as possible to the dots and that the person achieving the best recognition score would receive a $\$ 10$ reward. Both ratings and RTs were recorded.

\section{RESULTS}

\section{RT Task}

Because the two subgroups of subjects presented, in both conditions, with either SOC1 or SOC2, were trained identically, their RTs were combined for subsequent analyses. Figure 1 shows the average RTs obtained over the entire experiment, plotted separately for the two conditions. In order to analyze the data, we performed an analysis of variance (ANOVA) with blocks (15 levels) as a within-subjects variable and condition (2 levels) as a between-subjects variable. This analysis revealed significant effects of block $\left[F(14,308)=17.094, M S_{\mathrm{e}}=\right.$ $1,110.455, p<.0001]$ and condition $[F(1,22)=10.140$, $\left.M S_{\mathrm{e}}=40,145.88, p<.005\right]$. The interaction also reached significance $\left[F(14,308)=2.751, M S_{\mathrm{e}}=1,110.455, p<\right.$ $.001]$. However, closer examination of Figure 1 suggests that this significant interaction might in fact be attributed to the pattern obtained over the first three blocks of training. This impression was confirmed by an ANOVA conducted on the data after removal of the first three blocks $(F<1.2)$. Overall, subjects were faster in the RSI condition - a result that could be interpreted as resulting either from improved learning or from improved expression of the acquired knowledge.

Most importantly, suppression of the RSI did not prevent sequence learning. Indeed, RTs increased in both conditions when subjects were exposed to the transfer sequence on Block 13. This result was confirmed by another ANOVA with block (two levels, Blocks 12 and 13) as a within-subjects variable and condition (two levels) as a between-subjects variable. This analysis showed significant effects of block $\left[F(1,22)=35.144, M S_{\mathrm{e}}=2,382.12\right.$, 


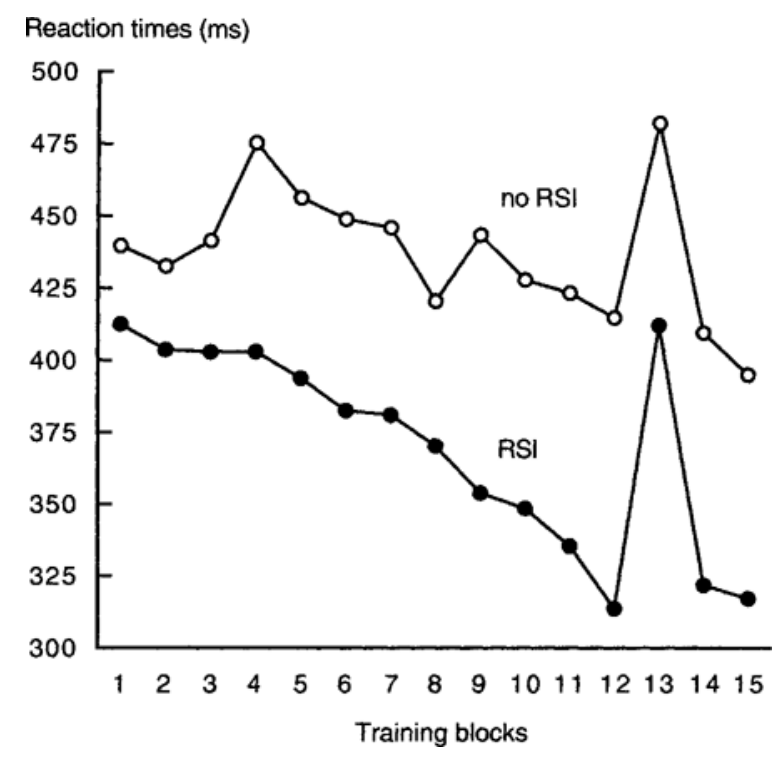

Figure 1. Mean reaction times for each training block, plotted separately for subjects trained with and without responsestimulus interval (RSI).

$p<.0001]$ and of condition $\left[F(1,22)=15.886, M S_{\mathrm{e}}=\right.$ $5,599.197, p<.001]$. RTs increased by $100 \mathrm{msec}$ in the RSI condition and by $68 \mathrm{msec}$ in the no-RSI condition, but the corresponding block $\times$ condition interaction failed to reach significance $(F<1.3)$. Presenting subjects with the training sequence anew on Blocks 14 and 15 allowed them to recover their pretransfer performance level. On the basis of these findings, we can thus conclude that subjects have learned the training sequence in both conditions. We now examine whether subjects in the RSI and no-RSI conditions differ in their ability to project their knowledge of the sequence in direct tests - that is, whether the corresponding knowledge is best described as implicit or explicit.

\section{Generation Tasks}

To measure generation performance, we computed the number of generated chunks of three elements that were part of the training sequence in both inclusion and exclusion tasks. Since the generated sequences were 96 trials long, the maximum number of correct chunks was 94 . To obtain inclusion and exclusion scores for each subject, we therefore divided the corresponding number of correct chunks by 94 . Since subjects were told not to produce repetitions, chance level was .33.

Figure 2 (left panel) shows average inclusion and exclusion scores for both conditions. An ANOVA with condition (RSI vs. no RSI) as a between-subjects variable and instructions (inclusion vs. exclusion) as a withinsubjects variable revealed a significant effect of instructions $\left[F(1,22)=11.977, M S_{\mathrm{e}}=0.018, p<.005\right]$ and a significant condition $\times$ instructions interaction $[F(1,22)=$ $\left.6.918, M S_{\mathrm{e}}=0.018, p<.05\right]$. Condition failed to reach significance. To find out whether generation performance reflects knowledge acquired during the SRT task, onetailed $t$ tests were used to compare generation scores with those expected at chance level.

Let us first examine the results of the inclusion task. Subjects' performance was above chance level in both conditions $[t(11)=3.62, p<.005$, and $t(11)=4.33, p<$ .0005 , for the RSI and the no-RSI conditions, respectively]. However, as shown by a planned comparison, the difference in inclusion performance between conditions was only marginally significant $\left[F(1,22)=3.511, M S_{\mathrm{e}}=\right.$ $0.028, p=.07]$. These results therefore appear to indicate that learning was in fact explicit in both conditions, because all subjects were able to project some of their knowledge about the sequence in the generation task. Similar associations between performance during the choice RT task and corresponding direct measures of sequence knowledge obtained in comparable settings (Perruchet \& Amorim, 1992; Shanks \& Johnstone, 1998, 1999) have been widely used to reject the idea that learning in such tasks is implicit.

However, a very different conclusion emerges when one also considers exclusion task performance. Indeed, while subjects trained in the RSI condition appear to have been capable of refraining from generating chunks of the training sequence, subjects trained in the no-RSI condition kept generating such chunks above chance level despite being specifically instructed not to do so [onetailed $t(11)=3.03, p<.01]$. Planned comparisons further revealed that the number of generated correct chunks decreased significantly from inclusion to exclusion instructions in the RSI condition $\left[F(1,22)=18.55, M S_{\mathrm{e}}=\right.$ $0.018, p<.0005]$ but not in the no-RSI condition $(p>.5)$. Hence, it appears that in contrast to RSI subjects, no-RSI subjects had no control over their knowledge of the sequence.

To further explore exclusion performance, we conducted an additional analysis, as follows: For each subject, we computed the number of generated chunks contained in the training sequence (the usual score) and also the number of chunks contained in the transfer sequence (on which subjects have not been trained). The rationale of this analysis is that if sequential knowledge has been acquired implicitly during the SRT task, then it should exert an automatic influence on performance during the generation task. In other words, under exclusion instructions, one would expect subjects to tend to produce the sequential regularities of the training sequence more often than those of some other appropriate control sequence (e.g., the transfer sequence) in spite of the exclusion instructions. This is exactly the pattern of results obtained in the exclusion task performed by subjects from the no-RSI condition, in which they tended to produce more chunks from the sequence on which they had been trained (37.7) than from the sequence on which they had not been trained (31.6) [one-tailed $t(11)=1.836, p<.05$ ]. In contrast, subjects should have had control over their explicit knowledge, and indeed, we observed that subjects from the RSI 


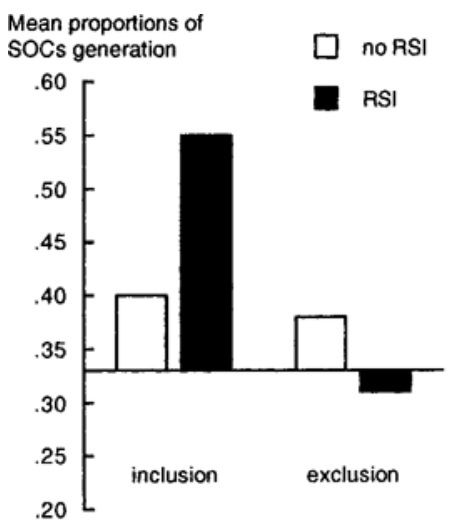

Instructions

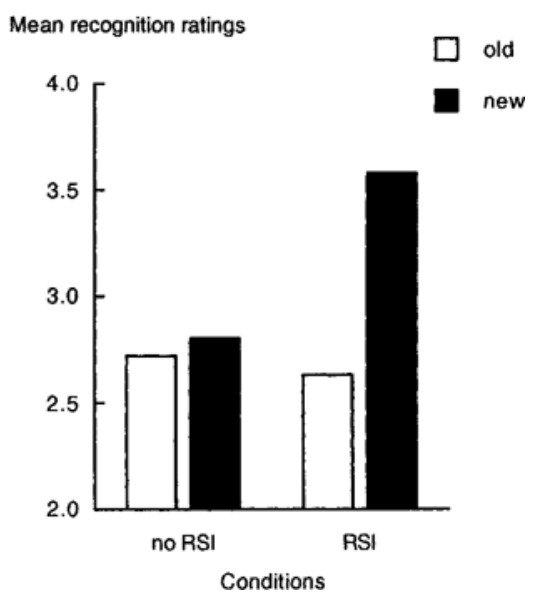

Figure 2. Left panel: Mean proportions of generated second order conditional transitions (SOCs) that were part of the training sequence, for both conditions, and under inclusion or exclusion instructions. Right panel: Mean recognition ratings given for the 24 test triplets. A high rating (between 4 and 6) is expected for a new sequence, and a low rating (between 1 and 3 ) is expected for an old sequence. condition were able to avoid producing more regular (30.4) than irregular (31.8) triplets when instructed to do so- that is, under exclusion conditions $(p>.3)$.

To summarize, learning in the no-RSI condition produced knowledge over which subjects had little control, whereas learning in the RSI condition produced knowledge that subjects could control. If control is taken to reflect availability to consciousness, one can conclude that learning in the no-RSI condition was unconscious. These conclusions are also confirmed by the results of the recognition task, which we describe in the next section.

\section{Recognition Task}

Subjects were asked to respond to sequences of three elements and to rate the extent to which they felt these sequences were familiar. Because subjects may tend to respond faster to familiar sequence fragments than to novel fragments, their ratings could reflect feelings of perceptual and motor fluency rather than explicit recollection (see Cohen \& Curran, 1993; Perruchet \& Amorim, 1992; Perruchet \& Gallego, 1993; Willingham, Greeley, $\&$ Bardone, 1993, for relevant discussion). To rule out this potential confound, we contrasted RTs elicited by the third element of old and new sequence fragments. ${ }^{3}$ An ANOVA with condition (RSI vs. no RSI) as a betweensubjects variable and sequence (old vs. new) as a withinsubjects variable applied to these data showed that neither factor nor their interaction reached significance (all $p \mathrm{~s}>$ .1). Given that RTs elicited by old and new sequence fragments do not differ in the recognition task, we can assume that perceptual motor fluency does not influence responses in this task-a result that replicates Shanks and Johnstone's (1999) data. Recognition scores can therefore be safely taken to reflect explicit recollection. ${ }^{4}$
Mean recognition ratings for both conditions and for both types of sequences are shown in Figure 2 (right panel; recall that high ratings correspond to judgments of novelty and are expected for new sequences). It is clear that subjects in the RSI condition were able to differentiate between old and new triplets. This is obviously not the case for subjects trained in the no-RSI condition. These observations were confirmed by an ANOVA performed on recognition ratings with condition (RSI vs. no RSI) as a between-subjects variable and sequence (old vs. new) as a within-subjects variable. This analysis revealed a significant effect of sequence $\left[F(1,22)=6.753, M S_{\mathrm{e}}=\right.$ $0.477, p<.05]$ and a significant condition $\times$ sequence interaction $\left[F(1,22)=4.738, M S_{\mathrm{e}}=0.477, p<.05\right]$. The effect of condition failed to reach significance. Planned comparisons further indicated that recognition ratings differed significantly between old and new sequences in the RSI condition only $\left[F(1,22)=11.402, M S_{\mathrm{e}}=0.477\right.$, $p<.005]$. These results are perfectly consistent with those obtained with the generation tasks, and again suggest that subjects trained in the no-RSI condition lacked explicit sequence knowledge.

\section{DISCUSSION}

The notion that sequence learning can occur implicitly has been previously rejected on the basis of observed associations between learning and performance on forcedchoice tasks used to assess explicit knowledge (e.g., Shanks \& Johnstone, 1998, 1999). In this study, while we confirmed the existence of such associations, we also suggested that they need not necessarily be interpreted as evidence that learning is explicit. Indeed, our results indicate that in a generation task performed under exclusion 
conditions, only subjects trained in the RSI condition were successful in performing the difficult exclusion task as instructed, while subjects trained in the no-RSI condition tended to keep producing chunks from the training sequence in spite of being specifically instructed not to do so. Taken together, these exclusion results can only be interpreted as indicating that in the no-RSI condition, generation performance under inclusion instructions is at least partly subtended by implicit sequence knowledge (Richardson-Klavehn, Gardiner, \& Java, 1996), and hence that the knowledge acquired during training on the SRT task must itself be at least partly unconscious. This conclusion is further supported by the inability of the no-RSI subjects to discriminate between novel and familiar sequence fragments in the recognition task. On the basis of these findings, we therefore conclude that sequence learning can proceed unconsciously. In the following, we would like to reflect on the methodological and theoretical implications of our study.

Methodologically, our findings demonstrate the importance of taking the contamination problem into account in implicit learning research: Even forced-choice testsotherwise widely taken to constitute the best available tests of explicit knowledge-turn out not to be immune from implicit influences, and therefore cannot be considered as being exclusively sensitive to explicit knowledge. Other methods designed to address the contamination problem in related fields have resulted in similar conclusions when applied to implicit learning. For instance, Dienes and colleagues (Dienes \& Altmann, 1997; Dienes et al.,1995; see also Shanks \& Johnstone, 1998), on the basis of Cheesman and Merikle's (1984) studies on subliminal perception, have proposed using subjective rather than objective criteria (i.e., performance on a forcedchoice task) to distinguish implicit from explicit learning. Subjective criteria, however, are prone to the same conceptual difficulties as verbal reports are, in that both rely on subjective self-assessments to determine the presence of some knowledge.

Another approach, based on Reingold and Merikle's framework (1988), was introduced by Jiménez et al. (1996), who proposed comparing the relative sensitivity of direct and indirect tasks in order to separately assess the influence of implicit versus explicit knowledge. The main limitation of this approach, however, is that the indirect task (the SRT task) must be assumed to be more sensitive than the direct task (the generation task) in order to demonstrate implicit influences (Toth, Reingold, \& Jacoby, 1994). However, it is possible that this condition might not always hold in the sequence learning paradigm (Shanks \& Johnstone, 1998, Experiment 3).

In the PDP, as used here, awareness is related to controlled responding. Within this framework, we have been able to demonstrate a qualitative dissociation between implicit and explicit sequence learning. Indeed, as our results indicate, increasing the RSI tends to exclusively improve explicit knowledge acquisition.
Turning now to the theoretical implications of our findings, we note that the PDP is a methodological framework that does not constitute, in and of itself, a process theory. Our findings therefore remain mute with respect to the existence of dissociable memory systems. However, our results suggest a functional dissociation between implicit and explicit learning and should therefore be useful in informing the development of computational models of implicit learning - an important limitation of which is that they have generally tended to remain agnostic about the implicit/explicit distinction.

Our interpretation of this functional dissociation is rooted in three central assumptions. The first is that explicit, conscious knowledge involves higher quality memory traces than does implicit knowledge. "Quality of representation," in this context, designates several properties of memory traces, such as their relative strength in the relevant information processing pathways, their distinctiveness, or their stability in time.

The second assumption is that memory traces continuously influence processing regardless of their quality. Thus, even weak traces, in our framework, while not available to conscious control, are nevertheless capable of influencing processing, but only through associative priming mechanisms - that is, in conjunction with additional contextual cues. Strong traces, in contrast, are available to conscious control in the sense that they can both trigger responses in the absence of other sources of constraints and be inhibited when required.

Our third assumption is that the development of higher quality representations takes time, both over training and during processing of a single event. Skill acquisition, for instance, involves the long-term progressive development of strong, high-quality memory traces based on early availability of weaker traces. Likewise, the extent to which memory traces can influence performance depends both on available processing time during a single trial and on asymptotic trace strength.

When applied to our data, this framework suggests the following interpretation: People trained with an RSI are given more opportunities to develop and link together high-quality memory traces than people in the no-RSI condition. Because awareness depends in part on the quality of stored memory traces, the former will tend to acquire more explicit knowledge than the latter. Importantly, no-RSI subjects do acquire relevant knowledge about the sequence — but in the form of weaker memory traces that are capable of influencing responses only when contextual information is simultaneously available. This knowledge can thus be expressed in the SRT task as well as in the generation tasks because in both cases responses can be determined on the joint basis of an external stimulus (self-generated in the case of the generation tasks, or produced by the experimental software in the SRT task) and the relevant memory traces. Because these traces are weak and because controlled processing (and hence awareness) requires high-quality traces to be available, their 
influence on performance remains undetected, and controlled responding is made difficult. The relevant sequential knowledge therefore cannot be inhibited when the generation task is performed under exclusion conditions. Similarly, during recognition, weak memory traces do not allow successful discrimination between old and novel sequences in the absence of perceptual and motor fluency, as was the case in our study.

In conclusion, we believe that our application of PDP to sequence learning provides a useful new tool for investigating the relationships between implicit and explicit learning, and that our results clearly demonstrate that awareness is not always necessary for learning to occur.

\section{REFERENCES}

Berry, D. C., \& Dienes, Z (1993). Implicit learning: Theoretical and empirical issues. Hillsdale, NJ: Erlbaum.

Buchner, A., Steffens, M. C., Erdfelder, E., \& Rothkegel, R. (1997). A multinomial model to assess fluency and recollection in a sequence learning task. Quarterly Journal of Experimental Psychology, 50A, 631-663.

Buchner, A., Steffens, M. C., \& Rothkegel, R. (1998). On the role of fragmentary knowledge in a sequence learning task. Quarterly Journal of Experimental Psychology, 51A, 251-281.

Cheesman, J., \& Merikle, P. M. (1984). Priming with and without awareness. Perception \& Psychophysics, 36, 387-395.

Clark, R. E., \& SQuire, L. R. (1998). Classical conditioning and brain systems: The role of awareness. Science, 280, 77-81.

Cleeremans, A., Destrebecqz, A., \& Boyer, M. (1998). Implicit learning: News from the front. Trends in Cognitive Sciences, 2, 406416.

Clegg, B. A., Digirolamo, G. J., \& Keele, S. W. (1998). Sequence learning. Trends in Cognitive Sciences, 2, 275-281.

Cohen, A., \& Curran, T. (1993). On tasks, knowledge, correlations, and dissociations: Comment on Perruchet and Amorim (1992). Journal of Experimental Psychology: Learning, Memory, \& Cognition, 19, 1431-1437.

Cohen, A., Ivry, R. I., \& Keele, S. W. (1990). Attention and structure in sequence learning. Journal of Experimental Psychology: Learning, Memory, \& Cognition, 16, 17-30.

Curran, T. (1995). On the neural mechanisms of sequence learning [On-line]. Psyche, 2. Available: http://psyche.csse.monash.edu.au/ v2/psyche-2-12-curran.html

Curran, T., \& Keele, S. W. (1993). Attentional and nonattentional forms of sequence learning. Journal of Experimental Psychology: Learning, Memory, \& Cognition, 19, 189-202.

Dienes, Z, \& Altmann, G. T. M. (1997). Transfer of implicit knowledge across domains: How implicit and how abstract? In D. C. Berry (Ed.), How implicit is implicit learning? (pp. 107-123). New York: Oxford University Press.

Dienes, Z., Altmann, G. T. M., Kwan, L., \& Goode, A. (1995). Unconscious knowledge of artificial grammars is applied strategically. Journal of Experimental Psychology: Learning, Memory, \& Cognition, 21, 1322-1338.

Frith, C., Perry, R. \& Lumer, E. (1999). The neural correlates of conscious experience: An experimental framework. Trends in Cognitive Sciences, 3, 105-114.

Goschke, T. (1997). Implicit learning and unconscious knowledge: Mental representation, computational mechanism, and brain structures. In K. Lambert \& D. Shanks (Eds.), Knowledge, concepts and categories (pp. 247-333). Hove, U.K.: Psychology Press.

Goschke, T. (1998). Implicit learning of perceptual and motor sequences: Evidence for independent learning systems. In M. A. Stadler \& P. A. Frensch (Eds.), Handbook of implicit learning (pp. 401-444). Thousand Oaks, CA: Sage.

JACOBY, L. L. (1991). A process dissociation framework: Separating au- tomatic from intentional uses of memory. Journal of Memory \& Language, 30, 513-541.

JACObY, L. L., Toth, J. P., \& Yonelinas, A. P. (1993). Separating conscious and unconscious influences on memory: Measuring recollection. Journal of Experimental Psychology: General, 122, 139-154.

Jiménez, L., Méndez, C., \& Cleeremans, A. (1996). Comparing direct and indirect measures of sequence learning. Journal of Experimental Psychology: Learning, Memory, \& Cognition, 22, 948-969.

Kinoshita, S. (2001). The role of involuntary aware memory in the implicit stem and fragment completion tasks: A selective review. Psychonomic Bulletin \& Review, 8, 58-69.

Lieberman, D. A., Sunnucks, W. L., \& Kirk, J. D. J. (1998). Reinforcement without awareness: I. Voice level. Quarterly Journal of Experimental Psychology, 51B, 301-316.

Merikle, P. M., \& Reingold, E. M. (1991). Comparing direct (explicit) and indirect (implicit) measures to study unconscious memory. Journal of Experimental Psychology: Learning, Memory, \& Cognition, 17, 224-233.

Neal, A., \& Hesketh, B. (1997). Episodic knowledge and implicit learning. Psychonomic Bulletin \& Review, 4, 24-37.

Nissen, M. J., \& Bullemer, P. (1987). Attentional requirement of learning: Evidence from performance measures. Cognitive Psychology, 19, 1-32.

Perruchet, P., \& Amorim, M. A. (1992). Conscious knowledge and changes in performance in sequence learning: Evidence against dissociation. Journal of Experimental Psychology: Learning, Memory, \& Cognition, 18, 785-800.

Perruchet, P., Bigand, E., \& Benoît-Gonin, F. (1997). The emergence of explicit knowledge during the early phase of learning in sequential reaction time. Psychological Research, 60, 4-14.

Perruchet,P., \& Gallego, J. (1993). Associations between conscious knowledge and performance in normal subjects: Reply to Cohen and Curran (1993) and Willingham, Greeley, and Bardone (1993). Journal of Experimental Psychology: Learning, Memory, \& Cognition, 19, 1438-1444.

REBER, A. (1993). Implicit learning and tacit knowledge: An essay on the cognitive unconscious. New York: Oxford University Press.

Reber, A., Allen, R, \& Reagan, S. (1985). Syntactical learning and judgment, still unconscious and still abstract. Journal of Experimental Psychology: General, 114, 17-24.

ReED, J., \& Johnson, P. (1994). Assessing implicit learning with indirect tests: Determining what is learned about sequence structure. Journal of Experimental Psychology: Learning, Memory, \& Cognition, 20, 585-594.

Reingold, E. M., \& Merikle, P. M. (1988). Using direct and indirect measures to study perception without awareness. Perception \& Psychophysics, 44, 563-575.

Richardson-KlaVehn, A., Gardiner, J. M., \& JaVA, I. (1996). Memory: Task dissociations, process dissociations and dissociations of consciousness. In G. Underwood (Ed.), Implicit cognition (pp. 85158). New York: Oxford University Press.

Shanks, D. R., \& Johnstone, T. (1998). Implicit knowledge in sequential learning tasks. In M. A. Stadler \& P. A. Frensch (Eds.), Handbook of implicit learning (pp. 533-572). Thousand Oaks, CA: Sage.

Shanks, D. R., \& Johnstone, T. (1999). Evaluating the relationship between explicit and implicit knowledge in a serial reaction time task. Journal of Experimental Psychology: Learning, Memory, \& Cognition, 25, 1435-1451.

Shanks, D. R, \& St. John, M. F. (1994). Characteristics of dissociable human learning systems. Behavioral \& Brain Sciences, 17, $367-$ 447.

Stadler, M. A., \& Roediger, H. L., III (1998). The question of awareness in research on implicit learning. In M. A. Stadler \& P. A. Frensch (Eds.), Handbook of implicit learning (pp. 105-132). Thousand Oaks, CA: Sage.

Toth, J. P., Reingold, E M., \& JACOBy, L. L. (1994). Toward a redefinition of implicit memory: Process dissociations following elaborative processing and self-generation. Journal of Experimental Psychology: Learning, Memory, \& Cognition, 20, 290-303. 
Willingham, D. B., Greeley, T., \& Bardone, A. M. (1993). Dissociation in a serial response time task using a recognition measure: Comment on Perruchet and Amorim (1992). Journal of Experimental Psychology: Learning, Memory, \& Cognition, 19, 1424-1430.

Willingham, D. B., Nissen, M. J., \& Bullemer, P. (1989). On the development of procedural knowledge. Journal of Experimental Psychology: Learning, Memory, \& Cognition, 15, 1047-1060.

\section{NOTES}

1. In a generation task, subjects are required to indicate the next element of the sequence rather than to react to the current one. In a recognition task, they are presented with small fragments of a sequence and asked to classify them as being part of the training pattern or not.

2 . The PDP has raised many controversies. However, these are mainly concerned with the specific measurement model used to obtain quanti- tative estimates of implicit and explicit influences on performance. Different models have been proposed that reflect the hypothetical relationship between both influences. In order to circumvent this issue, we based our adaptation of the PDP on the comparison between inclusion and exclusion performance only (see also Neal \& Hesketh, 1997).

3. Recall that two elements of temporal context are needed to predict the next location. Differences in RTs that specifically reflect sequence knowledge can therefore be observed only for the third element of each sequence fragment.

4. On the basis of previous results (Shanks \& Johnstone, 1999), it seems that more than three trials are needed to allow perceptual fluency to improve RTs in the recognition task.

(Manuscript received September 28, 1999; revision accepted for publication May 2, 2000.) 